\title{
A NEW $q$-LAGRANGE FORMULA AND SOME APPLICATIONS
}

\author{
CHRISTIAN KRATTENTHALER
}

\begin{abstract}
A new $q$-extension of the Lagrange-Bürmann expansion and related formulas are proved. Finally we give a method to find $q$-generalizations of Riordan's inverse relations.
\end{abstract}

1. Introduction. The Lagrange-Bürmann formula solves the problem of computing the coefficients $c_{k}$ in the expansion $g(z)=\sum_{k=0}^{\infty} c_{k} z^{k} / f^{k}(z)$, where $f(z)$ and $g(z)$ are given formal power series (fps) with $f(0) \neq 0$. In this paper we shall use a method introduced by Egorychev [2]. Consider $a(z)$ a Laurent series (Ls), then $\operatorname{coef}_{z}(a(z) d z)$ denotes the coefficient of $z^{-1}$ in $a(z)$. The two (equivalent) versions of the Lagrange formula can be rewritten as

$$
c_{n}=\underset{z}{\operatorname{coef}}\left(g(z) f^{n}(z)\left(1-z \frac{d f(z) / d z}{f(z)}\right) \frac{d z}{z^{n+1}}\right)
$$

or

$$
c_{n}=\frac{1}{n} \operatorname{coef}\left(\frac{d}{d z} g(z) f^{n}(z) \frac{d z}{z^{n}}\right) \text { for } n \geqslant 1 .
$$

Jackson [7] and Carlitz [1] found $q$-analogues in special cases connected with Abeland Laguerre polynomials, respectively. Garsia and Joni $[3,4]$ gave a very nice $q$-extension of (1.1), but it did not contain Jackson's special case. A q-extension containing both Jackson's and Carlitz's results is due to Hofbauer [6]. His results are special cases of Theorem 1 in this paper.

2. Definitions. Let $q$ be a fixed real number with $q \neq 0,1$. Then we define, as usual, $[\alpha]=\left(q^{\alpha}-1\right) /(q-1), \quad[n] !=[n] \cdot[n-1] \cdots[1],[0] !=1$ and $\left[\begin{array}{l}\alpha \\ n\end{array}\right]=$ $[\alpha][\alpha-1] \cdots[\alpha-n+1] /[n]$ !. We introduce the $q$-difference operator $D_{q}$ by

$$
D_{q} f(z)=(f(q z)-f(z)) /(q-1) z \text {. }
$$

Since $D_{q} z^{n}=[n] z^{n-1}, D_{q}$ is a linear operator on the set of Ls. If $a(z)$ is an Ls, the following property holds:

$$
\underset{z}{\operatorname{coef}}\left(D_{q} a(z) d z\right)=0
$$

Received by the editors July 12, 1982.

1980 Mathematics Subject Classification. Primary 05A19, 05A15.

Key words and phrases. $q$-Lagrange formula, inverse relations, $q$-exponential function. 
The $q$-exponential function $e_{q}(z)=\sum_{k=0}^{\infty} z^{k} /[k]$ ! satisfies the differential equation $D_{q} e_{q}(z)=e_{q}(z)$, which is equivalent to

$$
e_{q}(q z)=(1+(q-1) z) e_{q}(z) .
$$

Finally, we define

$$
p_{\alpha}(1, z)=\frac{e_{q}\left(q^{\alpha} z /(1-q)\right)}{e_{q}(z /(1-q))} .
$$

Use of (2.1) and (2.3) gives $D_{q} p_{\alpha}(1, z)=-[\alpha] p_{\alpha-1}(1, q z)$ and by iteration $D_{q}^{k} p_{\alpha}(1, z)$ $=(-1)^{k} q^{\left(\frac{k}{2}\right)} p_{\alpha-k}\left(1, q^{k} z\right)[\alpha][\alpha-1] \cdots[\alpha-k+1]$. Therefore, we have

$$
p_{\alpha}(1, z)=\sum_{k=0}^{\infty} \frac{\left.D_{q}^{k} p_{\alpha}(1, t)\right|_{t=0}}{[k] !} z^{k}=\sum_{k=0}^{\infty}(-1)^{k} q^{\left(\frac{k}{2}\right)}\left[\begin{array}{l}
\alpha \\
k
\end{array}\right] z^{k} .
$$

3. The Lagrange formula. Hofbauer's idea is based on the observation that

$$
\frac{d}{d z} f^{n}(z)=n \cdot \frac{f^{\prime}(z)}{f(z)} \cdot f^{n}(z) \text {. }
$$

This leads to

Definition 1. The fps $\varphi_{\alpha}(z), \alpha \in \mathbf{R}$ (= real numbers), are called $q$-powers, if there is a fixed fps $\varphi(z)$ such that $\varphi_{\alpha}(0) \neq 0$ for all $\alpha$ and

$$
D_{q} \varphi_{\alpha}(z)=[\alpha] \varphi(z) \varphi_{\alpha}(z) \text {. }
$$

EXAMPLE 1. Let us suppose $a, b \in \mathbf{R}$, and $m$ is a positive integer, then $e_{q^{m}}\left((a[\alpha]+b) z^{m}\right) / e_{q^{m}}\left(b z^{m}\right)$ are $q$-powers corresponding to

$$
\varphi(z)=\frac{a[m] z^{m-1}}{1+\left(q^{m}-1\right) b z^{m}} .
$$

To see this we only have to use (2.1) and (2.3), which leads to

$$
D_{q}\left(\frac{e_{q^{m}}\left((a[\alpha]+b) z^{m}\right)}{e_{q^{m}}\left(b z^{m}\right)}\right)=\frac{a[\alpha][m] z^{m-1}}{\left(1+\left(q^{m}-1\right) b z^{m}\right)} \frac{e_{q^{m}}\left((a[\alpha]+b) z^{m}\right)}{e_{q^{m}}\left(b z^{m}\right)} .
$$

LEMMA 1. Let $\varphi_{\alpha}(z)$ and $\phi_{\alpha}(z)$ be q-powers corresponding to $\varphi(z)$ and $\phi(z)$, respectively. Take $\lambda, \mu \in \mathbf{R}$, then

$$
\begin{aligned}
& \underset{z}{\operatorname{coef}}\left(\frac{\varphi_{n+\lambda}(z) / \phi_{n-\mu}(q z)}{\varphi_{k+\lambda}(q z) / \phi_{-k-\mu}(z)}\right. \\
& \left.\cdot \frac{\left(1-z \varphi(z)-z \phi(z)+z^{2} \varphi(z) \phi(z)\left(1-q^{\lambda-\mu}\right)\right)}{z^{n-k+1}} d z\right)=\delta_{n k}
\end{aligned}
$$

where $\delta_{n k}$ is the Kronecker delta.

Proof. Observe that (3.1) is equivalent to

$$
\varphi_{\alpha}(q z)=\left(1+\left(q^{\alpha}-1\right) z \varphi(z)\right) \varphi_{\alpha}(z)
$$


and $\phi_{\alpha}$ the same. By using (2.1) and (3.3) we get

$$
\begin{gathered}
D_{q}\left(\frac{\varphi_{n+\lambda}(z) / \phi_{-n-\mu}(z)}{\varphi_{k+\lambda}(z) / \phi_{-k-\mu}(z) \cdot z^{n-k}}\right)=\frac{\varphi_{n+\lambda}(z) / \phi_{-n-\mu}(q z)}{\varphi_{k+\lambda}(q z) / \phi_{-k-\mu}(z)} \frac{1}{(q-1) z^{n-k+1} q^{n-k}} \\
\cdot\left[\left(1+\left(q^{n+\lambda}-1\right) z \varphi(z)\right)\left(1+\left(q^{-k-\mu}-1\right) z \phi(z)\right)\right. \\
\left.-q^{n-k}\left(1+\left(q^{-n-\mu}-1\right) z \phi(z)\right)\left(1+\left(q^{k+\lambda}-1\right) z \varphi(z)\right)\right] \\
=-\frac{[n-k]}{q^{n-k}} \frac{\varphi_{n+\lambda}(z) / \phi_{-n-\mu}(q z)}{\varphi_{k+\lambda}(q z) / \phi_{-k-\mu}(z)} \\
\cdot \frac{\left(1-z \varphi(z)-z \phi(z)+z^{2} \varphi(z) \phi(z)\left(1-q^{\lambda-\mu}\right)\right)}{z^{n-k+1}} .
\end{gathered}
$$

If $n \neq k$ we have proved (3.2) by remembering (2.2). The case $n=k$ can be evaluated directly.

We now obtain the $q$-extensions of (1.1) and (1.2) as easy consequences of this lemma.

THEOREM 1. With the assumptions of Lemma 1 and $g(z)$ an fps we have:

(A) If

$$
g(z)=\sum_{k=0}^{\infty} c_{k} \frac{z^{k}}{\varphi_{k+\lambda}(q z) / \phi_{-k-\mu}(z)}
$$

then

$$
c_{n}=\underset{z}{\operatorname{coef}}\left(g(z) \frac{\varphi_{n+\lambda}(z)}{\phi_{-n-\mu(q z)}}\left(1-z \varphi(z)-z \phi(z)+z^{2} \varphi(z) \phi(z)\left(1-q^{\lambda-\mu}\right)\right) \frac{d z}{z^{n+1}}\right) .
$$

(B) If

$$
g(z)=g(0)+\sum_{k=1}^{\infty} c_{k} \frac{z^{k}}{\varphi_{k}(z) / \phi_{-k}(z)}
$$

then

$$
c_{n}=\frac{1}{[n]} \underset{z}{\operatorname{coef}}\left(D_{q}(g(z)) \frac{\varphi_{n}(z)}{\phi_{-n}(q z)} \frac{d z}{z^{n}}\right)
$$

Proof. (A) is obvious. Concerning (B), evaluate

$$
\begin{aligned}
D_{q}\left(\frac{z^{k}}{\varphi_{k}(z) / \phi_{-k}(z)}\right) & =\frac{z^{k}}{\varphi_{k}(q z) / \phi_{-k}(z)} \frac{1}{(q-1) z} \\
& \cdot\left(q^{k}\left(1+\left(q^{-k}-1\right) z \phi(z)\right)-\left(1+\left(q^{k}-1\right) z \varphi(z)\right)\right) \\
& =[k] \frac{z^{k-1}}{\varphi_{k}(q z) / \phi_{-k}(z)}(1-z \varphi(z)-z \phi(z)) .
\end{aligned}
$$


Therefore,

$$
\begin{aligned}
& \underset{z}{\operatorname{coef}}\left(D_{q}\left(g(z) \frac{\varphi_{n}(z)}{\phi_{-n}(q z)} \frac{d z}{z^{n}}\right)\right. \\
& \quad=\operatorname{coef}\left(\sum_{k=1}^{\infty}[k] c_{k} \frac{\varphi_{n}(z) / \phi_{-n}(q z)}{\varphi_{k}(q z) / \phi_{-k}(z)} \frac{(1-z \varphi(z)-z \phi(z))}{z^{n-k+1}} d z\right)=[n] c_{n}
\end{aligned}
$$

by Lemma 1 , setting $\lambda=\mu=0$.

\section{Examples.}

Example 2 (JACKson's Special CASE). By setting $b=0$ and $m=1$ in Example 1, we see that $e_{q}(a[\alpha] z)$ are $q$-powers corresponding to $a$. Use of Theorem 1 gives: If

$$
g(z)=\sum_{k=0}^{\infty} c_{k} \frac{z^{k}}{e_{q}(a q[k+\lambda] z)},
$$

then

$$
c_{n}=\underset{z}{\operatorname{coef}}\left(g(z) e_{q}(a[n+\lambda] z)(1-a z) \frac{d z}{z^{n+1}}\right)
$$

and if

$$
g(z)=g(0)+\sum_{k=1}^{\infty} c_{k} \frac{z^{k}}{e_{q}(a[k] z)}
$$

then

$$
c_{n}=\frac{1}{[n]} \underset{z}{\operatorname{coef}}\left(D_{q}(g(z)) e_{q}(a[n] z) \frac{d z}{z^{n}}\right) .
$$

Example 3 (CARlitz's Special CASe). Take $a=-1, b=1 /(1-q)$ and $m=1$. Because of (2.4) we get: $p_{\alpha}(1, z)$ are $q$-powers corresponding to $-1 /(1-z)$. Finally, simple calculations show that by Theorem 1 we have: If

$$
g(z)=\sum_{k=0}^{\infty} c_{k} \frac{z^{k}}{p_{k+\lambda}(1, q z)},
$$

then

$$
c_{n}=\underset{z}{\operatorname{coef}}\left(g(z) p_{n+\lambda-1}(1, q z) \frac{d z}{z^{n+1}}\right)
$$

and if

$$
g(z)=g(0)+\sum_{k=1}^{\infty} c_{k} \frac{z^{k}}{p_{k}(1, z)},
$$

then

$$
c_{n}=\frac{1}{[n]} \underset{z}{\operatorname{coef}}\left(D_{q}(g(z)) p_{n}(1, z) \frac{d z}{z^{n}}\right) .
$$


EXAMPLE 4. In Theorem 1, take $\varphi_{\alpha}(z)=\phi_{\alpha}(z)=p_{\alpha}(1, z)$ and $\mu=0$. Again we avoid the calculations, which lead to: If

$$
g(z)=\sum_{k=0}^{\infty} c_{k} \frac{z^{k}}{p_{2 k+\lambda+1}\left(1, q^{-k} z\right)}
$$

then

$$
c_{n}=\operatorname{coef}_{z}\left(g(z) p_{2 n+\lambda-1}\left(1, q^{-n+1} z\right)\left(1-q^{\lambda} z^{2}\right) \frac{d z}{z^{n+1}}\right)
$$

and if

$$
g(z)=g(0)+\sum_{k=1}^{\infty} c_{k} \frac{z^{k}}{p_{2 k}\left(1, q^{-k} z\right)},
$$

then

$$
c_{n}=\frac{1}{[n]} \underset{z}{\operatorname{coef}}\left(D_{q}(g(z)) p_{2 n-1}\left(1, q^{-n+1} z\right)(1-z) \frac{d z}{z^{n}}\right)
$$

In [5] I. Gessel and D. Stanton obtain these three examples as special cases of a theorem about $q$-Lagrange inversion. It seems that with the exception of a few examples this theorem cannot be derived by our theory. It would be very interesting to find the connections between these results.

5. A theorem about inverse relations. In [2] Egorychev gave a method to prove all the inverse relations of Riordan [8]. To find $q$-inverse relations we use a more general result based upon the same idea.

THEOREM 2. Let $\left(g_{n}(z)\right)_{n=0}^{\infty},\left(G_{k}(z)\right)_{k=0}^{\infty},\left(h_{n}(z)\right)_{n=0}^{\infty}$ and $\left(H_{k}(z)\right)_{k=0}^{\infty}$ be sequences of fps with

$$
\underset{z}{\operatorname{coef}}\left(\frac{g_{n}(z)}{G_{k}(z)} \frac{d z}{z^{n-k+1}}\right)=\operatorname{coef}\left(\frac{h_{n}(z)}{H_{k}(z)} \frac{d z}{z^{n-k+1}}\right)=\delta_{n k} .
$$

If $\left(\alpha_{n}\right)_{n=0}^{\infty}$ and $\left(\beta_{n}\right)_{n=0}^{\infty}$ are sequences of real numbers different from zero and $f(z)$ is an fps with $f(0) \neq 0$, then $a_{n}=\sum_{k=0}^{n} c_{n k} b_{k}$ holds with

$$
c_{n k}=\frac{\beta_{k}}{\alpha_{n}} \operatorname{coef}_{z}\left(f(z) \frac{g_{n}(z)}{H_{k}(z)} \frac{d z}{z^{n-k+1}}\right)
$$

if and only if $b_{n}=\sum_{k=0}^{n} d_{n k} a_{k}$ with

$$
d_{n k}=\frac{\alpha_{k}}{\beta_{n}} \operatorname{coef}\left(f(z)^{-1} \frac{h_{n}(z)}{G_{k}(z)} \frac{d z}{z^{n-k+1}}\right) .
$$

Proof. It is sufficient to prove only one implication; the other follows by symmetry. We show “ $\Rightarrow$ ":

$$
\alpha_{n} a_{n}=\underset{z}{\operatorname{coef}}\left(\sum_{k=0}^{\infty} \alpha_{k} a_{k} \frac{z^{k}}{G_{k}(z)} \frac{g_{n}(z)}{z^{n+1}} d z\right)
$$


by (5.1). On the other hand, we have

$$
\begin{aligned}
\alpha_{n} a_{n} & =\alpha_{n} \sum_{k=0}^{n} c_{n k} b_{k}=\sum_{k=0}^{n} \alpha_{n} b_{k} \cdot \frac{\beta_{k}}{\alpha_{n}} \operatorname{coef}\left(f(z) \frac{g_{n}(z)}{H_{k}(z)} \frac{d z}{z^{n-k+1}}\right) \\
& =\underset{z}{\operatorname{coef}}\left(f(z) \sum_{k=0}^{\infty} b_{k} \beta_{k} \frac{z^{k}}{H_{k}(z)} \frac{g_{n}(z)}{z^{n+1}} d z\right) .
\end{aligned}
$$

Since (5.2) and (5.3) hold for every nonnegative integer $n$, the following equation is true:

$$
f(z) \sum_{k=0}^{\infty} b_{k} \beta_{k} \frac{z^{k}}{H_{k}(z)}=\sum_{k=0}^{\infty} \alpha_{k} a_{k} \frac{z^{k}}{G_{k}(z)}
$$

Use of (5.1) and (5.4) gives

$$
\begin{aligned}
\beta_{n} b_{n} & =\operatorname{coef}_{z}\left(\sum_{k=0}^{\infty} \beta_{k} b_{k} \frac{z^{k}}{H_{k}(z)} \frac{h_{n}(z)}{z^{n+1}} d z\right) \\
& =\operatorname{coef}_{z}\left(f(z)^{-1} \sum_{k=0}^{\infty} \alpha_{k} a_{k} \frac{z^{k}}{G_{k}(z)} \frac{h_{n}(z)}{z^{n+1}} d z\right) \\
& =\sum_{k=0}^{n} \alpha_{k} a_{k} \operatorname{coef}\left(f(z)^{-1} \frac{h_{n}(z)}{G_{k}(z)} \frac{d z}{z^{n-k+1}}\right) .
\end{aligned}
$$

Note that the last step essentially needs the condition $f(0) \neq 0$. Division by $\beta_{n}$ completes the proof.

Obviously, Lemma 1 gives many examples for the pairs $g_{n}(z), G_{k}(z)$ and $h_{n}(z)$, $H_{k}(z)$ by using Example 1. Indeed, it is possible to find explicit $q$-analogues of Riordan's inverse relations to Chebyshev-, Legendre- or Abel-type. Some simple examples are listed below.

$$
\begin{aligned}
& a_{n}=\sum_{k=0}^{n}(-1)^{n-k}\left[\begin{array}{c}
n+p \\
n-k
\end{array}\right] b_{k} \Leftrightarrow b_{n}=\sum_{k=0}^{n} q^{\left(\frac{n-k}{2}\right)}\left[\begin{array}{c}
n+p \\
n-k
\end{array}\right] a_{k}, \\
& a_{n}=\sum_{k=0}^{[n / 2]}(-1)^{k}\left[\begin{array}{c}
n+p \\
k
\end{array}\right] b_{n-2 k} \Leftrightarrow b_{n}=\sum_{k=0}^{[n / 2]} q^{\left(\frac{k}{2}\right)}\left[\begin{array}{c}
n+p-k \\
k
\end{array}\right] \frac{[n+p]}{[n+p-k]} a_{n-2 k}, \\
& a_{n}=\sum_{k=0}^{n}(-1)^{n-k}\left[\begin{array}{c}
2 n+p \\
n-k
\end{array}\right] b_{k} \Leftrightarrow b_{n}=\sum_{k=0}^{n} q^{\left(\frac{n-k}{2}\right)}\left[\begin{array}{c}
n+p+k \\
n-k
\end{array}\right] \frac{[2 n+p]}{[n+p+k]} a_{k}, \\
& a_{n}=\sum_{k=0}^{n}(-1)^{n-k}\left[\begin{array}{l}
n \\
k
\end{array}\right][n+p]^{n-k} b_{k} \Leftrightarrow b_{n}=\sum_{k=0}^{n} q^{\left(\frac{n-k}{2}\right)}\left[\begin{array}{l}
n \\
k
\end{array}\right][n+p][k+p]^{n-k-1} a_{k} .
\end{aligned}
$$




\section{REFERENCES}

1. L. Carlitz, Some q-expansion formulas, Glas. Mat. Ser. III 8 (1973), 205-214.

2. G. P. Egorychev, Integral representation and computation of combinatorial sums, Novosibirsk. 1977.

3. A. M. Garsia and S. A. Joni, Composition sequences, Comm. Algebra 8(1980), 1195-1266.

4. A. M. Garsia, A q-analogoue of the Lagrange inversion formula, Houston J. Math. 7 (1981), 205-237.

5. I. Gessel and D. Stanton, Applications of q-Lagrange inversion to basic hypergeometric series, Trans. Amer. Math. Soc. 277 (1983), 173-203.

6. J. Hofbauer, A q-analog of the Lagrange expansion, Arch. Math. (to appear).

7. F. H. Jackson, A q-generalization of Abel's series, Rend. Circ. Mat. Palermo 29 (1910), 340-346.

8. J. Riordan, Combinatorial identities, Wiley, New York, 1968.

Institut fUr Matematik, A-1090 Wien, Strudlhofgasse 4, Austria 